\title{
Choriocarcinoma Masquerading as Lung Abscess or Lung Cancer: A Case with Atypical Imaging Findings
}

\author{
Qianqian Gu',* \\ Shuangquan Yan ${ }^{1, *}$ \\ Jiang $\operatorname{Lin}^{2}$ \\ Xiaomai Wu (D) \\ Lanxi Chen ${ }^{3}$ \\ Meifu $\mathrm{Gan}^{3}$ \\ Huarong Luo ${ }^{3}$ \\ Dongqing $\mathrm{Lv}^{\prime}$ \\ Ling Lin'
}

'Department of Respiratory Medicine, Taizhou Hospital of Zhejiang Province affiliated to Wenzhou Medical University, Taizhou, 317000, People's Republic of China; ${ }^{2}$ Department of Thoracic Surgery, Taizhou Hospital of Zhejiang Province affiliated to Wenzhou Medical University, Taizhou, 317000, People's Republic of China; ${ }^{3}$ Department of Pathology, Taizhou Hospital of Zhejiang Province affiliated to Wenzhou Medical University, Taizhou, 317000, People's Republic of China

*These authors contributed equally to this work

Correspondence: Dongqing Lv; Ling Lin Taizhou Hospital of Zhejiang Province affiliated to Wenzhou Medical University, I50 Ximen Street, Linhai, 317000,

People's Republic of China

Email Ivdq@enzemed.com;

linling@enzemed.com
Background: Choriocarcinoma is a highly malignant trophoblastic tumor. However, the awareness surrounding its atypical clinical presentation is insufficient. The presence of a solitary lung lesion without uterine lesions often leads to misdiagnosis or missed diagnosis, which in turn causes delayed treatment or even multiple metastases throughout the body.

Case Presentation: We present the case of a 36-year-old female patient who was misdiagnosed with a lung abscess and received suboptimal anti-infective treatment. She then underwent left upper lobectomy and was misdiagnosed with lung cancer by abscess incision and drainage in thoracic surgery, however, the results after pleural effusion removal were suboptimal. During this time a breast nodule was found, and a large segment of the right breast was excised and misdiagnosed as breast cancer but was finally diagnosed as choriocarcinoma with multiple metastases of lung and breast. Multiple metastases were also detected in the head, liver, kidney, and bones. The patient underwent multiple adjuvant chemotherapies. The blood $\beta$-hCG level gradually declined to normal. When we reported this case, that is, seven months after the diagnosis, the patient was still alive, and the disease was stable without progress.

Conclusion: Choriocarcinoma with a solitary lung lesion as the first presentation and no lesions in the uterus is clinically rare. This may lead to a delay in diagnosis due to poor awareness of the disease and the appearance of multiple metastases throughout the body. Clinicians should be more aware of choriocarcinoma with an atypical presentation to reduce misdiagnosis and missed diagnosis.

Keywords: gestational trophoblastic tumor, choriocarcinoma, lung, misdiagnosis, $\beta$-hCG

\section{Introduction}

Gestational trophoblastic neoplasia (GTN), including invasive hydatidiform mole, choriocarcinoma, placental site trophoblastic tumor (PSTT), and epithelioid trophoblastic tumor (ETT) predispose to early onset of hematogenous metastasis, and the lungs are the most common site of metastasis (70-90\% of cases). ${ }^{1}$

Choriocarcinoma is a type of gestational trophoblastic tumor that is highly malignant accompanied by abnormal, uncontrolled proliferation of trophoblastic stem cells. It comprises mononuclear cytotrophoblasts and human chorionic gonadotropin (hCG)-producing multinucleated syncytiotrophoblasts that invade blood vessels at an early stage and are highly prone to multiple widespread metastases throughout the body, including organs such as the lungs, brain, liver, pelvis, vagina, spleen, intestines, and kidneys. ${ }^{2}$ Brain metastases occur in $11-28 \%$ 
of patients and often indicate a poor disease prognosis. ${ }^{3,4}$ The typical lung metastasis of choriocarcinoma mainly shows multiple metastatic foci, but choriocarcinoma with solitary lung lesions appear first without uterine lesions; these are rarely detected in clinical practice, and can easily cause misdiagnosis or missed diagnosis.

We present the case of a 36-year-old woman, whose diagnosis and treatment were tortuous, and was finally diagnosed as metastatic choriocarcinoma.

\section{Case Presentation}

A 36-year-old female patient who complained of an intermittent nocturnal cough for one week was admitted to the Department of Respiratory Medicine (Taizhou Hospital of Zhejiang Province, Linhai, China) on 2019-09-12. She had no history of dyspnea, chest pain, hemoptysis, or fever.

On 2019-09-17, diagnostic chest enhanced computed tomography (CT) revealed nodules in the upper lobe of the left lung (Figure 1A). After giving moxifloxacin anti-infection treatment for more than 10 days, her cough improved. The 2019-10-21 review chest CT revealed a tongue segment mass in the left lung, suggestive of malignancy, and a micronodule in the upper lobe of the right lung (Figure 1B). The lung tumor markers in blood were negative. The bronchoscopy was negative. Pulmonary examination revealed clear breath sounds in both lungs and slightly lower breath sounds in the left lower lung. The patient underwent $\mathrm{CT}$ guided lung biopsy. No malignant cells were detected by the ThinPrep cytologic test. The cryptococcal capsular antigen measurement, G-test, GM test, and IgG test for Aspergillus fumigatus antibodies were all negative. A positron emission tomography-CT (PETCT) scan on 2019-10-30 suggested an infective lesion in the lingual segment of the left lung.

The patient was treated with ceftriaxone needle 2.0 intravenous drip once daily for two weeks but she was still coughing both white sputum and dark red bloody sputum. On 2019-11-10, fever appeared, with the highest body temperature at $39.4{ }^{\circ} \mathrm{C}$. She also experienced leftsided chest pain, with chest tightness after activity that was slightly relieved by rest. On 2019-11-11, chest CT showed lesions occupying the space of the upper lobe tongue segment of the left lung, in addition, some inflammation and fibrous foci were also observed in the two lungs with tiny nodules in the anterior segment of the upper lobe of the right lung, and some effusion on both sides (Figure 1C). No improvement was observed after the administration of moxifloxacin (0.4 intravenous drip qd) for one week. On 2019-11-17, the patient began coughing

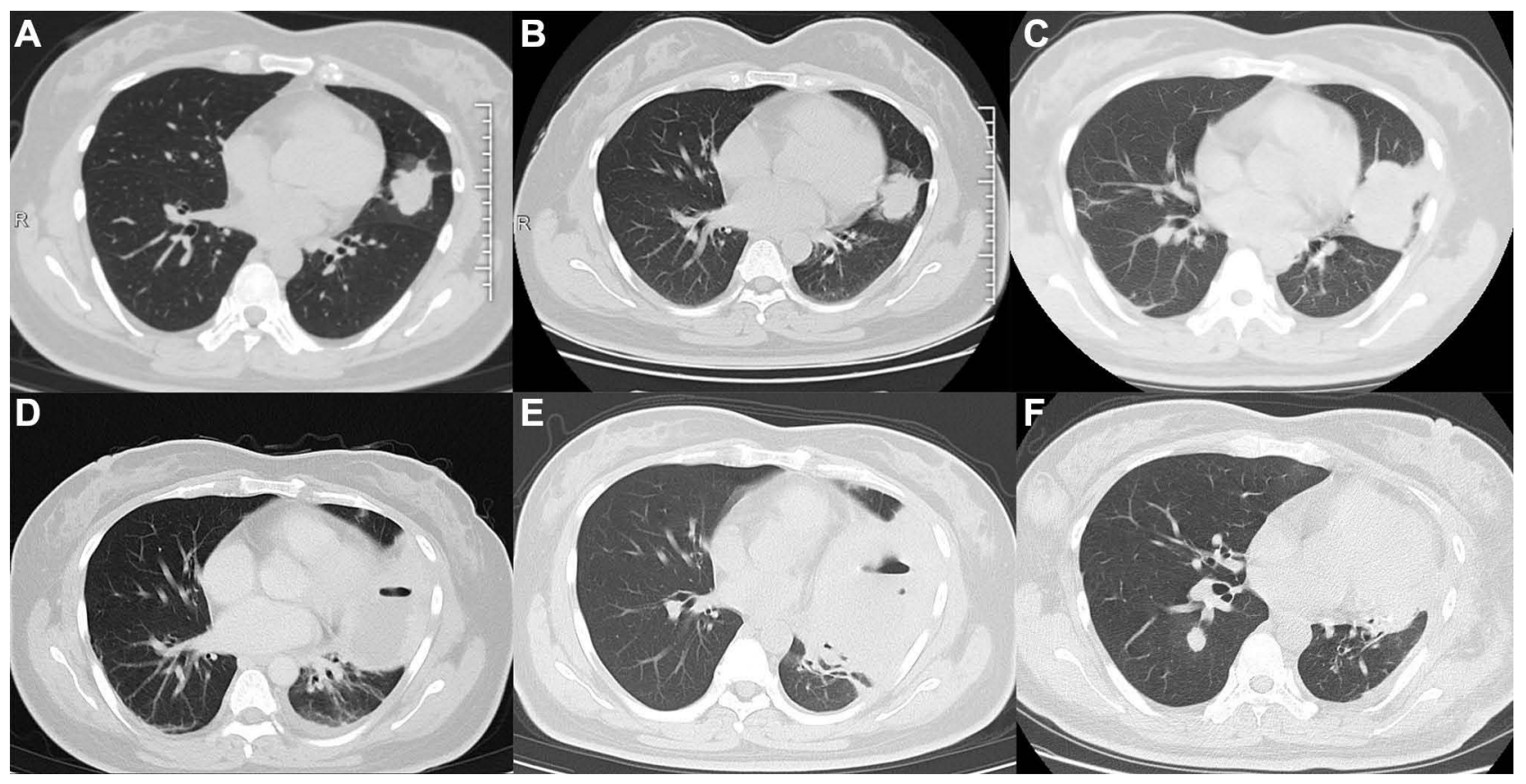

Figure I Chest computed tomography (CT) findings. (A) Chest CT showing a round nodule-like shadow in the left upper lobe, with a clear boundary and shallow lobule of about $2.5 \mathrm{~cm}$ diameter. (B) After anti-infection treatment, the chest CT showed a high-density shadow in the tongue segment of the left lung, with a size of about $3.2 \mathrm{~cm} *$ $2.7 \mathrm{~cm}$, likely to be neoplastic. (C) Chest CT showing a $5.3 \mathrm{~cm} * 3.5 \mathrm{~cm}$ tumor mass in the lingual segment of the left upper lobe. (D) The lesion in the left upper lobe was about $9.1 \mathrm{~cm} * 6.7 \mathrm{~cm}$, with bubbles and liquid dark areas. After enhanced CT, the lesion showed circular enhancement. (E) Chest CT showing a mass of $8.8 \mathrm{~cm} * 8.0 \mathrm{~cm}$ in the upper lobe of the left lung, containing gas-liquid plane. (F) Chest CT showing a small nodular high-density shadow in the lower lobe of right lung with clear boundary and $1.2 \mathrm{~cm}$ diameter. 
purulent yellow sputum. Bronchoalveolar lavage was given and bronchoscopy revealed congestion and edema of the mucosa at the mouth of the left lingual duct, and no obvious active bleeding (Figure 2). The test results of the lavage fluid were as follows: cell classification: total cells, 8660.0 cells/ $\mu \mathrm{L}$; phagocytes, $13 \%$; neutrophils, $85 \%$; lymphocytes, 1\%; eosinophils, 1\%. Cryptococcal antigen detection, mycobacterial species identification (ChIP method), lavage fluid culture, and fungal culture of the lavage fluid were all negative. Examination of the alveolar lavage fluid by next-generation metagene sequencing reported relative abundances of $83.5 \%$ Prevotella spp. and $9.5 \%$ Streptococcus spp. Anti-infection treatment of anaerobe incorporation was started by administering imipenemestatin (1.0 g intravenous drip Q8H) combined with clindamycin (0.6 intravenous drip q12h) was started. By 2019-11-23, the patient's body temperature had returned to normal. Enhanced CT of the chest showed obstruction in the upper lobe of the left lung. A lung abscess was considered, with obstructive pneumonia, and there was slight effusion in the thorax on both sides (the left more anterior) (Figure 1D). On 2019-11-28, the patient underwent thoracic surgery and CT-guided left lung abscess puncture by placing a drainage tube, but drainage of the effusion was not effective. On 2019-12-02, she underwent a thoracoscopic left lung abscess incision and drainage together with pleural effusion clearance and pus wall biopsy. Postoperative pathological diagnosis of the left lung indicated fibrocystic mural-like tissue with abscess formation. On 2019-12-20, the patient was prescribed faropenem tablets ( 0.2 oral thrice daily) as an anti-infective treatment for 10 days. By 2019-12-30, a small amount of bright red bloody liquid appeared and the cough was still frequent with the production of more yellow sputum. On 2020-01-02, a review of chest CT showed the presence of a mass shadow in the upper lobe of the left lung, with visible air and fluid. The right pleural effusion shadow was more absorbed, and the left pleural effusion shadow appeared more anterior (Figure 1E). The patient received meropenem (1 $\mathrm{g}$ intravenous drip Q8H) anti-infective treatment.

On 2020-01-08, the patient underwent left upper lobectomy along with lower lung repair. The postoperative pathological assessment showed a poorly differentiated non-small cell carcinoma occupying the upper lobe of the left lung, consistent with large cell neuroendocrine carcinoma, with abscess formation, and reactive hyperplasia of 18 lymph nodes (Figure 3A). Immunohistochemical results were: thyroid transcription factor 1 (TTF-1) (-), napsin A (-), p40 (minority +), cytokeratin (CK) 5/6 (-), synaptophysin (syn) (-), cluster of differentiation 56 (CD56) (+), CK (+), p63 (minority + ), neuron-specific enolase (NSE) (-), chromogranin A (CGA) (-), Ki-67 (approximately 90\%+). Genetic testing revealed no mutations on amplification refractory mutation system (ARMS). The PD-L1 level was 20\%.

On 2020-02-04, the patient was admitted to the Department of Oncology Surgery (Taizhou Hospital of Zhejiang Province, Linhai, China) due to a painless palpable mass in the right breast that had been present for two days (Figure 4). Chest CT showed left residual pneumonia with segmental atelectasis (less anterior), left pleural effusion, and small nodules in the right lung (Figure 1F). On 202002-05, the patient underwent right breast large segment resection and sentinel lymph node biopsy. Routine pathology on 2020-02-12 showed poorly differentiated carcinoma of the right breast with extensive hemorrhagic necrosis (considering the high possibility of breast origin) with reactive hyperplasia of sentinel lymph nodes (two stations) (Figure 3B). Immunohistochemical findings were: estrogen

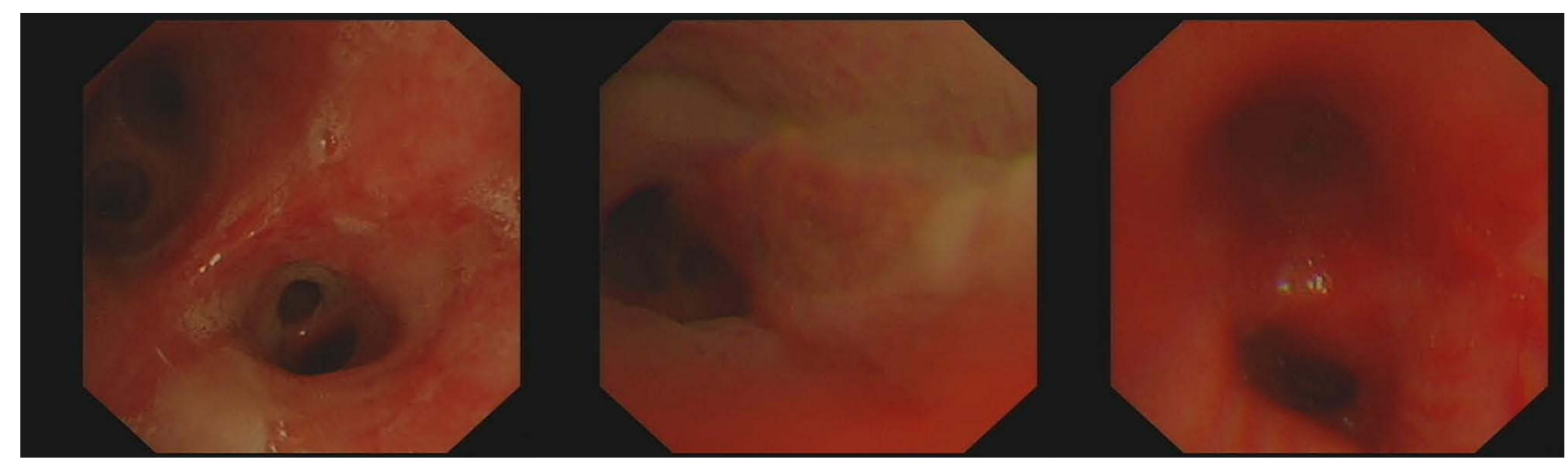

Figure 2 Bronchoscopy revealing congestion and edema of the mucosa at the mouth of the left lingual duct. 


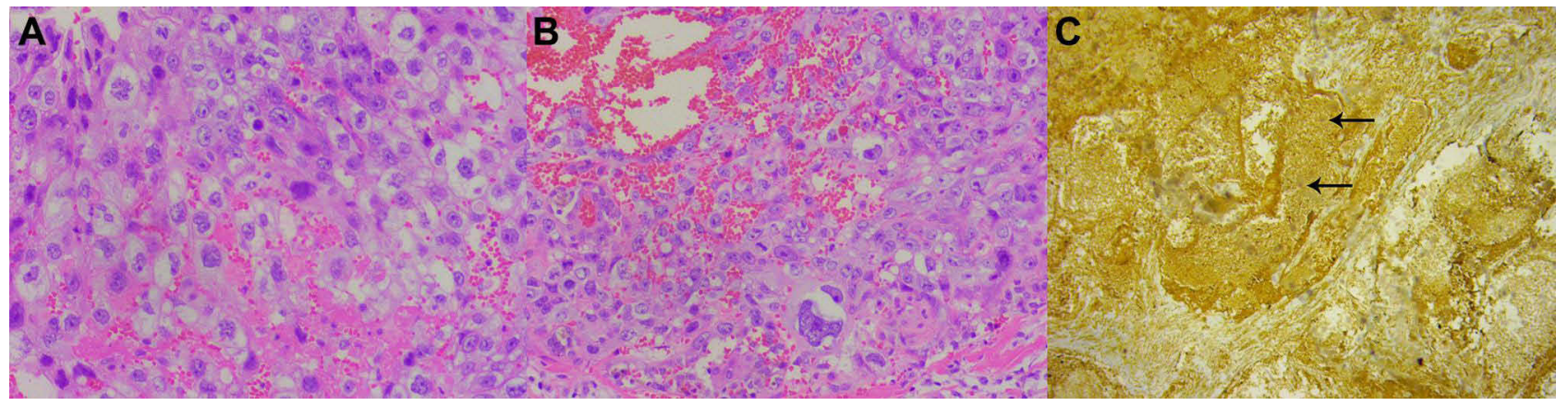

Figure 3 Hematoxylin and eosin staining of a tumor section $(\times 200)$. (A) Pathological examination under light microscopy, showing a poorly differentiated non-small cell carcinoma occupying the space of the upper lobe of the left lung. (B) Pathological examination under light microscopy, showing a poorly differentiated carcinoma of the right breast with extensive hemorrhagic necrosis. (C) Immunohistochemical staining for $\beta$-hCG (positive in foci).

receptor $(\mathrm{ER})(-)$, progesterone receptor $(\mathrm{PR})(-)$, receptor tyrosine-protein kinase erbB-2 (CerbB-2) $(1+)$, GATA binding protein $3($ GATA3) $(+)$, CK $(+)$, vimentin $(-)$, P63 (focal +), TTF-1 (-), syn (-), CGA (-), CD56 (focal +), Ki-67 (90\% $+)$, mammaglobin (-), gross cystic disease fluid protein 15 (GCDFP-15) (scattered weak + ), CK7 (+).

Pathology consultation at the Second Affiliated Hospital of Zhejiang University revealed parabronchial large cell carcinoma of the upper left lung, with morphology, hematoma formation, and immunophenotype similar to those of the carcinoma in the right breast. These findings, combined with immunohistochemical results, suggest that lung lesions are caused by metastasis of breast cancer to the lungs. The vasculature showed no tumor thrombus or nerve invasion, and the bronchial margins were

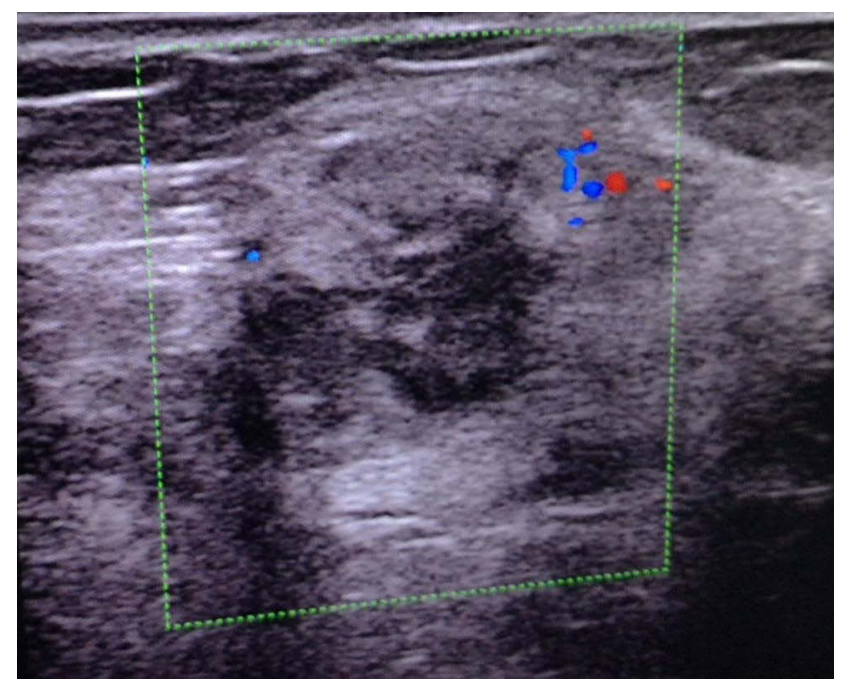

Figure 4 Breast ultrasound showing hypoechoic mass in the right breast (BI-RADS 4a) (green box). negative. Eighteen lymph nodes were examined, all with negative results, and no tumor metastasis was seen.

Immunohistochemical analysis conducted at a second hospital showed strongly positive GATA $(3+)$, weakly positive ER (-) and PR (1 +), and positive CerbB-2 (2 + ), indicative of invasive carcinoma and hematoma formation in the right breast at grade 3 , and intraductal carcinoma with no tumor thrombus in the vasculature and negative resection margins. Two sentinel lymph nodes were negative. Further immunohistochemical results were: smooth muscle actin $(+)$, CD10 (+), CD31 foci $(+)$, and ERG (-).

On 2020-03-09, a review of the chest CT showed multiple metastases in both lungs, left residual pneumonia with segmental atelectasis, a little left pleural effusion, and left pleural hypertrophic adhesion (Figure 5A). In a review of the medical history and pathological results on 2020$3-9$, the blood $\beta-\mathrm{hCG}$ reading was $21,136.00 \mathrm{mIU} / \mathrm{mL}$. Complementary breast tumor tissue immunohistochemistry showed hCG $(+)$ (Figure 3C). The final diagnosis was poorly differentiated carcinoma of the right breast (consistent with choriocarcinoma) with reactive hyperplasia of the sentinel lymph nodes (two lymph nodes). Complementary lung tumor tissue immunohistochemistry showed hCG $(+)$. The final diagnosis was poorly differentiated carcinoma of the upper left lung (consistent with choriocarcinoma) with reactive hyperplasia of lymph nodes (18 cases).

On 2020-03-10, Positron emission tomography (PET) revealed 2-deoxy-2- $\left[{ }^{18} \mathrm{~F}\right]$ fluoro-d-glucose uptake in the pulmonary nodules, liver, left kidney, left erector spinae and quadratus psoas, right humerus and ilium, and tubercle of the right frontal lobe and occipital lobe, which suggested the presence of multiple metastases in these areas. 


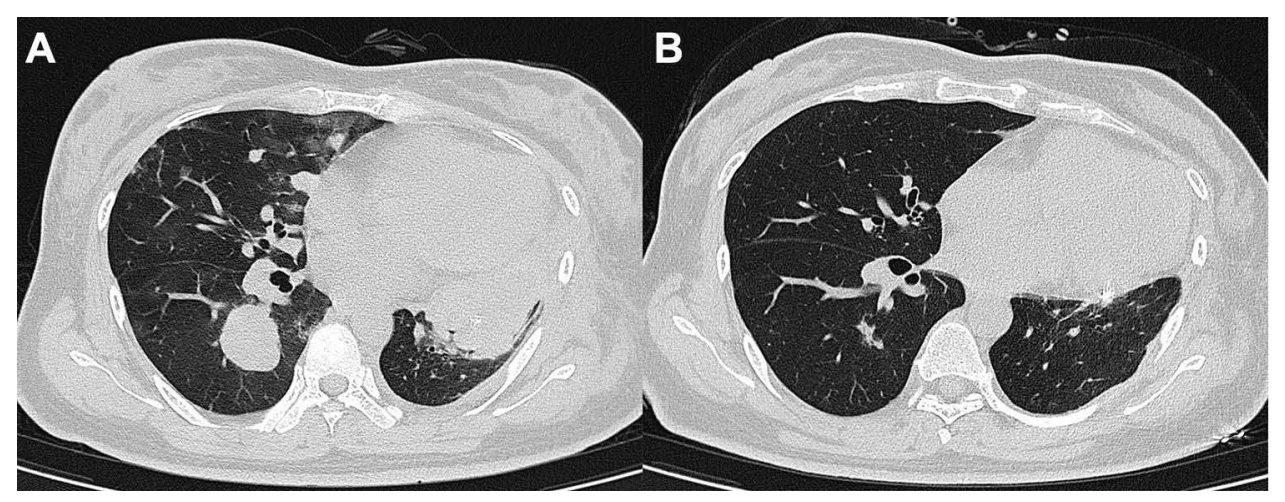

Figure 5 Chest computed tomography (CT) before and after chemotherapy. (A) Chest CT showing multiple nodules and masses in both lungs, the larger of which was about $5.7 \mathrm{~cm} * 3.4 \mathrm{~cm}$. (B) Chest CT after chemotherapy showing tumors smaller than those before.

On 2020-3-11, after a discussion with multidisciplinary experts, we considered a diagnosis of choriocarcinoma (stage IV, FIGO score 17), with multiple metastases to the lung, breast, liver, kidney, and bones. The patient was transferred to the gynecology department and underwent curettage on the same day. No intraepithelial lesions or malignant cells were found on the ThinPrep cytologic test. The Cervista ThinPrep cytological test for high-risk HPV DNA was negative, indicating no high-risk HPV infection. The pathology of the uterine cavity showed endometrial tissue with stromal decidualization. Microscopic examination showed round and tubular endometrial glands, several of which were dilated. The epithelial cells of the glands showed evidence of secretion and the stromal cells showed decidual changes. A detailed medical history was taken of the patient's menstrual cycle, indicating cycles of 23-37 days and menstrual periods of five days. There was one miscarriage in 2014, induced labor in 2015 because the fetus had Down's syndrome, and eight oocyte retrievals in 2019 followed by thirdgeneration IVF that was unsuccessful. On 2020-3-12, a lumbar puncture was performed and the $\beta-h C G$ in the cerebrospinal fluid was determined to be $114.80 \mathrm{mIU} / \mathrm{mL}$.

On 2020-3-12, line EMA-CO scheme chemotherapy was initiated (VP16 etoposide $0.16 \mathrm{~g}$ static drop + MTX methotrexate $0.32 \mathrm{~g}+$ ACTD actinomycin $0.5 \mathrm{mg}$ static drop D1 chemotherapy, etoposide $0.16 \mathrm{~g}$ static drop + methotrexate $0.16 \mathrm{~g}+$ actinomycin $0.5 \mathrm{mg}$ static drop D2 chemotherapy, vindesine $3.2 \mathrm{mg}$ static drop + cyclophosphamide $0.9 \mathrm{~g}$ static drop D8 chemotherapy). Intrathecal methotrexate $(15 \mathrm{mg})$ via lumbar puncture was administered on 2020-03-13, -16, and -20th. To reduce methotrexate toxicity, she was given leucovorin $15 \mathrm{mg}$ intramuscular $\mathrm{q} 12 \mathrm{~h} * 4$ times from $2020-03-13$ to -15 . During the treatment, the patient developed 2 degrees of myelosuppression, 1 degree of dermatitis, and right epistaxis after the chemotherapy.

On 2020-04-01, the patient received two cycles of lowdose EP (etoposide $0.16 \mathrm{~g}$ static drip + cisplatin $32 \mathrm{mg}$ static drip QW) induction chemotherapy. On 2020-04-20, the EMA-EP regimen (VP16 etoposide $0.16 \mathrm{~g}$ static drop + MTX methotrexate $0.16 \mathrm{~g}$ static drop + MTX methotrexate $0.32 \mathrm{~g}$ static drop + ACTD actinomycin $0.5 \mathrm{mg}$ static drop D1 chemotherapy, etoposide $0.16 \mathrm{~g}$ static drop + actinomycin $0.5 \mathrm{mg}$ static drop D2 chemotherapy, etoposide 0.25 g static drop + cisplatin $120 \mathrm{mg}$ static drop D8 chemotherapy) was started for salvage chemotherapy. A total of 10 cycles of the EMA-EP regimen chemotherapy were administered to the patient by 2020-11-04.

By $2020-08-02$, the blood $\beta$-hCG was reduced to normal and the general condition was good. The 2020-10-22 review chest CT scan showed that the shadow of the two lung nodules was significantly reduced compared with previous scans, and the shadows of the two lung patches were absorbed (Figure 5B). The 2020-10-24 review head MRI, compared to that on 2020.03.11 (Figure 6A), showed remission of the metastases of the frontal lobe on both sides and of the occipital lobe on the right side compared to that on the anterior lobe (Figure 6B).

\section{Discussion}

Choriocarcinoma occurs primarily in women of reproductive age with marked ethnic and geographic variations. The incidence of choriocarcinoma is approximately $1 /$ 40,000 in Europe and North America. ${ }^{5}$ It may be classified as gestational or non-gestational choriocarcinoma.

Gestational choriocarcinoma is often secondary to a normal or abnormal pregnancy, including hydatidiform mole, abortion, term birth, or ectopic pregnancy. The 


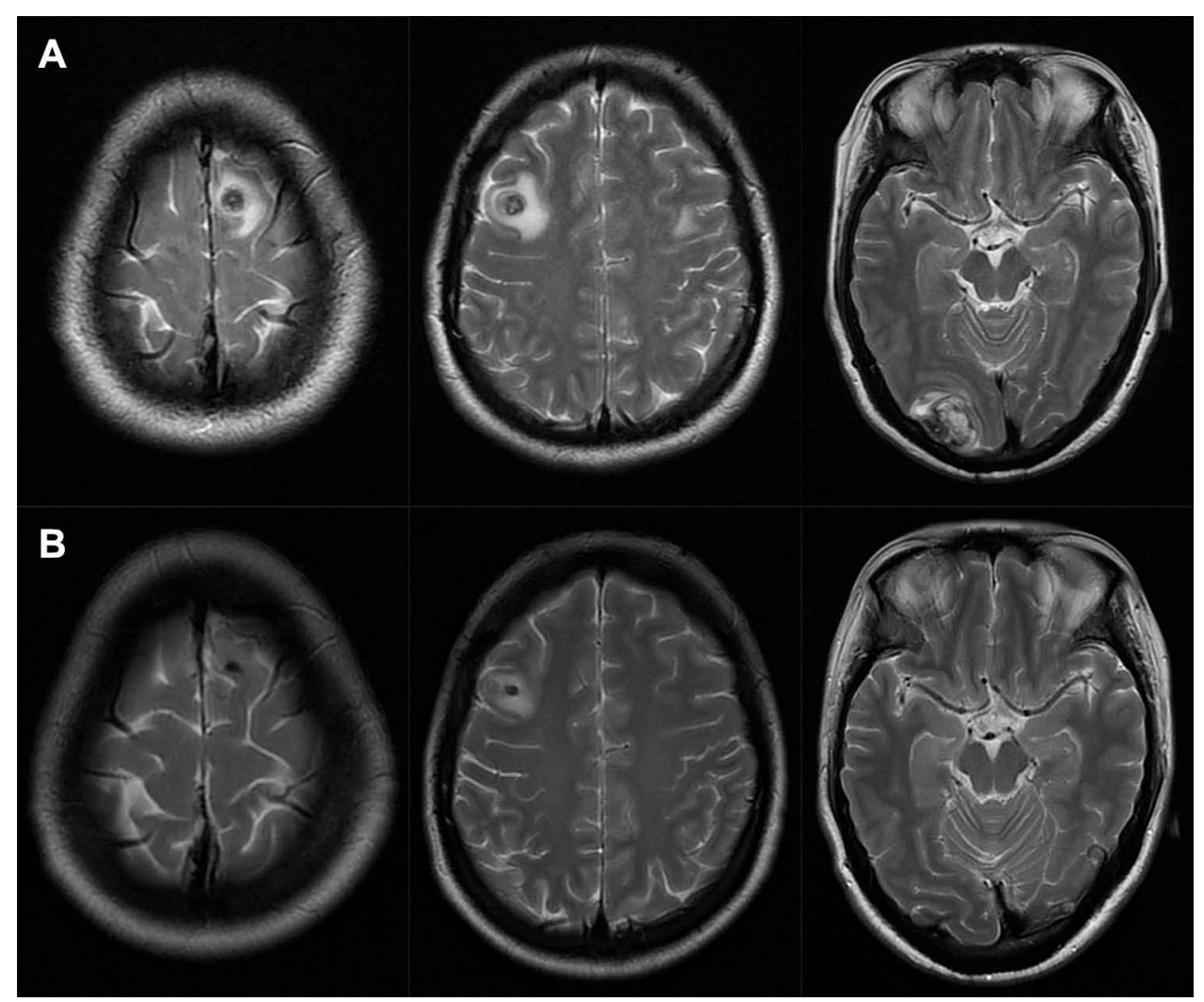

Figure 6 Brain magnetic resonance imaging (MRI) before and after chemotherapy. (A) Brain MRI before chemotherapy shows multiple nodules in bilateral frontal lobe and right occipital lobe, suggesting metastasis with a little bleeding. (B) After chemotherapy, brain MRI shows that bilateral frontal lobe and right occipital lobe nodules were smaller than before, and the peripheral edema was relieved.

secondary intervals are variable, with some occurring at the beginning of pregnancy, and intervals of up to 30 years have also been reported. ${ }^{6}$ The development of the tumor may be related to the last pregnancy when trophoblasts reach the lungs through the bloodstream and lie dormant while maintaining their reproductive capacity, reactivating when the body immunity is reduced or affected by other factors, and developing into choriocarcinoma within the lungs. ${ }^{6,7}$ Our patient had had one miscarriage five years before the onset and induction of labor four years before, with a history of IVF oocyte retrieval in the year of onset. Therefore, metastatic gestational choriocarcinoma was diagnosed, and it can be seen that a detailed reproductive history is very important for the diagnosis of this disease.

Choriocarcinoma mostly presents with persistent irregular bleeding from the vagina. This case was asymptomatic and had undergone whole-body PET-CT twice during the entire procedure showing no abnormal features of the uterine adnexa. The first symptoms were related to the pulmonary metastases, with hemoptysis, chest tightness, and chest pain as the main clinical manifestations.
However, in this type of situation, the first physician is likely to be a specialist in respiratory medicine, it is possible to not inquire about the previous gynecological history or not obtain sufficient details, resulting in either a misdiagnosis or missed diagnosis.

The typical findings of choriocarcinoma pulmonary metastasis imaging are dominated by multiple lesions, such as multiple shadow films, multiple shadow nodules, and multiple lumps, most often in the lung periphery. ${ }^{8}$ Atypical imaging findings include solitary cavitations and solitary nodules. A single, giant, block-type lung metastasis of choriocarcinoma is rare, and a single intrapulmonary metastatic lesion, showing relatively clear contours and smooth margins can have a shallow lobulation sign. The contours of primary lung cancers tend to be spiculated with fine spiculations and a lobulated sign. In our patient, initial imaging showed a single mass shadow with liquefactive necrosis in the middle of the lesion. This finding, combined with the respiratory symptoms, led to the diagnosis of the lung abscess, a relatively common condition. Even the pathological results after the first operation did not indicate a definite diagnosis. 
A review of the literature shows the existence of three hypotheses for the presence of solitary lung lesions in choriocarcinoma. First, pulmonary choriocarcinoma may represent metastasis from an unidentified trophoblastic disease that may have undergone spontaneous regression leaving only a uterine scar; the so-called "burn-out" phenomenon is a unique and specific feature of choriocarcinoma that is likely to metastasize before the primary lesion is discovered. ${ }^{9,10}$ Second, choriocarcinoma may arise from pulmonary epithelium retained primordial germ cells that have migrated abnormally during embryonic development or pulmonary embolism by trophoblastic tissue related to the gestational events. ${ }^{11}$ Third, trophoblastic differentiation or metaplasia may occur in lung cancer, leading to pure choriocarcinoma or a mixed tumor associated with lung cancer differentiation. This last hypothesis is supported by thyroid transcription factor-1 immunoreactivity in the nuclei of the syncytiotrophoblast-like cells observed in malignant lung tumors. ${ }^{12}$

All choriocarcinomas are accompanied by the production of hCG, which is detectable in the serum or urine. In cases such as this, if a detailed obstetric and gynecological history can be acquired, the possibility of early choriocarcinoma is considered, and perfect the blood hCG test, the diagnosis is not difficult, nor does it make the patient undergo multiple operations.

Microscopically, choriocarcinoma tumor tissue is composed of poorly differentiated trophoblast-like and syncytiotrophoblast-like tumor cells with marked cellular atypia and nuclear karyorrhexis. These two types of cells were mixed and arranged in a nest or cord-like shape, and obvious hemorrhagic necrosis was observed in the cancer tissue and surrounding normal tissues. The cancer cells did not form villi and vesicle-like structures. Pathologically, in this patient, the heterotypic nuclei were large, hyperchromatic, irregular, and arranged in a nest shape, suggestive of poorly differentiated carcinoma. Because the specimens were from the lung and breast, and not in the uterus, choriocarcinoma was not initially considered before further verification by the hCG test. After the immunohistochemical results showed TTF-1 (-) and napsina (-), primary lung tumors were excluded. The P40 (minority + ), CK $(+)$, and p63 (minority + ) results indicated epithelial origin, but P40 and p63 were only weakly positive. In addition, NSE (-), syn (-), CGA (-), and CD56 were positive; these are only considered in common diseases, and large cell neuroendocrine carcinoma was initially considered. After the second operation of breast cancer, the pathological sections of the breast were immunohistochemically analyzed and the results were CerbB-2 $(1+)$, ER (-), PR (-), GATA3 (+), gcdfp15 (scattered weak + ), and mammaglobin (-). Based on immunohistochemical results, the possibility of poorly differentiated breast cancer was considered. For GATA3 positive, we only identified only breast and lung cancers without divergent thinking and identifying other diseases. The pathological sections were sent to the Second Affiliated Hospital of Zhejiang University for pathological consultation and were further added to the immunohistochemical markers. GATA3 (3+), ER (-), PR (1+), C-erB-2 (2+), and most of the immunohistochemical markers of breast cancer were positive, so they were misdiagnosed as invasive breast cancer.

The initial manifestation of this case was of the lung origin, the initial manifestation of lung symptoms, no typical clinical manifestations of choriocarcinoma, coupled with the lack of understanding of the disease, and it is too rare in clinical, lack of experience, leading to the diagnosis of this case tortuous and difficult. Due to the general lack of knowledge on clinical departments involved in respiratory, extra-thoracic, and thyroid breast surgery, as well as radiology and pathology departments, should consider this disease to avoid delays in diagnosis. It is, therefore, necessary to improve the awareness of clinical and related ancillary departments towards this disease to prevent misdiagnosis and missed diagnoses. When single or multiple lesions appear on lung imaging and the primary site is unknown, care should be taken to screen for possible metastases of gynecological disease, as approximately $50 \%$ of choriocarcinomas are secondary to complete hydatidiform mole, $25 \%$ occur after a normal pregnancy, and another $25 \%$ are secondary to spontaneous abortion or ectopic pregnancy. ${ }^{5}$ For these high-risk patients, we need to track their blood HCG concentration. The taking of a detailed history and routine gynecological examination, as well as hCG testing, often enables an early diagnosis of the disease. Unfortunately, there are still about $25 \%$ of women who are unable to make a preventive diagnosis (normal pregnancy). Nevertheless, even if the preventive follow-up is correctly carried out in the gynecology departments, an appropriate therapeutic response would still have to be found.

\section{Data Sharing Statement}

The datasets supporting the conclusions of this case are included in the article. 


\section{Ethical Approval and Consent to Participate}

The study obtained the approval of the ethics committee of Taizhou Hospital of Zhejiang Province affiliated to Wenzhou Medical University (approval number: K20201101). Written consent to participate was provided by the patient for the present research.

\section{Patient Consent for Publication}

Informed consent was obtained from the patient for the publication of the case and any accompanying images. Copies of the written consent are available for review by the Editor-in-Chief of this journal.

\section{Funding}

This work was supported by the Medical Science and Technology Project Foundation of Zhejiang Province of China (2015KYB439) and Scientific Research Fund of Taizhou Science and Technology Agency (1601KY05, 21ywa24 and 1801KY19).

\section{Disclosure}

The authors report no conflicts of interest in this work.

\section{References}

1. Lurain JR. Gestational trophoblastic disease II: classification and management of gestational trophoblastic neoplasia. Am $J$ Obstet Gynecol. 2011;204(1):11-18. doi:10.1016/j.ajog.2010.06.072
2. Seckl MJ, Sebire NJ, Berkowitz RS. Gestational trophoblastic disease. Lancet. 2010;376:717-729. doi:10.1016/S0140-6736(10) 60280-2

3. Piura E, Piura B. Brain metastases from gestational trophoblastic neoplasia: review of pertinent literature. Eur J Gynaecol Oncol. 2014;35:359-367.

4. Weir B, MacDonald N, Mielke B. Intracranial vascular complications of choriocarcinoma. Neurosurgery. 1978;2:138-142. doi:10.1227/ 00006123-197803000-00012

5. Lurain JR. Gestational trophoblastic disease I: epidemiology, pathology, clinical presentation and diagnosis of gestational trophoblastic disease, and management of hydatidiform mole. Am J Obstet Gynecol. 2010;203:531-539. doi:10.1016/j.ajog.2010.06.073

6. Guo N, Yin R, Li Q, Song L, Wang D. Postmenopausal choriocarcinoma: a rare case report and review of the literature. Menopause. 2018;25(2):1-3.

7. Mukherjee U, Thakur V, Katiyar D, Pendharkar D, Pendharkar D. Uterine choriocarcinoma in a postmenopausal woman. Med Oncol. 2006;23:301-303. doi:10.1385/MO:23:2:301

8. Suprasert P, Eua-Throngchit J, Srisomboon J, Charoenkwan ZK, Phongnarisorn $\mathrm{C}$. Radiologic features and treatment outcomes of pulmonary metastasis in gestational trophoblastic neoplasia. $J$ Med Assoc Thai. 2005;88(7):875-880.

9. Rossi G, Valli D, Rivasi F, Longo L. Does primary pulmonary choriocarcinoma really exist? Chest. 2003;123:313. doi:10.1378/ chest.123.1.313

10. Berthod G, Bouzourene H, Pachinger C, Peters S. Solitary choriocarcinoma in the lung. $J$ Thorac Oncol. 2010;5:574-575. doi:10.1097/JTO.0b013e3181 cbf372

11. Ibi T, Hirai K, Bessho R, Kawamoto M, Koizumi K, Shimizu K. Choriocarcinoma of the lung: report of a case. Gen Thorac Cardiovasc Surg. 2012;60:377-380. doi:10.1007/s11748-012-0009-3

12. Chen F, Tatsumi A, Numoto S. Combined choriocarcinoma and adenocarcinoma of the lung occurring in a man: case report and review of the literature. Cancer. 2001;91:123. doi:10.1002/10970142(20010101)91:1<123::AID-CNCR16>3.0.CO;2-3
OncoTargets and Therapy

\section{Publish your work in this journal}

OncoTargets and Therapy is an international, peer-reviewed, open access journal focusing on the pathological basis of all cancers, potential targets for therapy and treatment protocols employed to improve the management of cancer patients. The journal also focuses on the impact of management programs and new therapeutic

\section{Dovepress}

agents and protocols on patient perspectives such as quality of life, adherence and satisfaction. The manuscript management system is completely online and includes a very quick and fair peer-review system, which is all easy to use. Visit http://www.dovepress.com/ testimonials.php to read real quotes from published authors. 